\title{
Constructing a P2P-Based High Performance Computing Platform*
}

\author{
Hai Jin, Fei Luo, Xiaofei Liao, Qin Zhang, and Hao Zhang
}

\author{
Cluster and Grid Computing Laboratory, \\ Huazhong University of Science and Technology, Wuhan, 430074, China \\ \{hjin, luofeiren, xfliao, qzhang, haozhang\}@hust.edu.cn
}

\begin{abstract}
The construction for a P2P-based high performance computing platform (P2HP) is presented to address parallel problems in this paper. $\mathrm{P} 2 \mathrm{HP}$ utilizes idle computers in the Internet with great scalability to form an enormous computing capability for scientific supercomputing and volunteers form autonomous unstructured P2P network domains. The configuration of P2HP is easy and a programming model is provided. Its applications involve a large range of problems, and a benchmark is applied to evaluate its performance.
\end{abstract}

\section{Introduction}

Peer-to-Peer (P2P) fashion can construct supercomputers far beyond the power of any current computing center, which make HPC much less expensive and much more accessible than clusters [1]. Because P2P addresses failure for dynamics, personal computers (PCs) in the Internet are suitable to participant into $\mathrm{P} 2 \mathrm{P}$ computing. There are countless PCs which are idle most of time. The collection of them in P2P systems has potentially enormous computing power.

Currently, most P2P-based computing systems belong to special organizations, and they are just used to solve one scientific or commercial high throughput problem. The characteristic of HPC is more instantaneous than that of high throughput computing (HTC), which makes them more tight constraints in HPC systems than in HTC [2].

A P2P-based HPC platform, P2HP, is depicted to assemble idle Internet resources in this paper. P2HP is constructed as a 3-layer network architecture, where volunteers form autonomous unstructured $\mathrm{P} 2 \mathrm{P}$ domains. The computing power increases with the rise of volunteers number. It is easily configured, and the management cost is distributed into autonomous domains, which brings the advantage that P2HP fits various environments and users. A one-sided message passing (OMP) [3] programming model is provided for users, which makes P2HP can work on a variety of problems, especially infinite workpile applications with deadlines [4].

The rest of the paper is organized as follows. Related works are reviewed in section 2. The system architecture of P2HP is outlined in section 3, and its design issues are described in section 4 . To test its high performance characteristics, sequence alignment in bioinformatics is applied as a benchmark in section 5. Finally, a conclusion is drawn in section 6.

"This paper is supported by National Science Foundation under grant 60433040, China CNGI project under grant CNGI-04-12-2A and CNGI-04-12-1D. 


\section{Related Works}

There are many P2P-based computing systems designed to harness idle cycles of Internet-connected workstations [5], which are also known as "public-resource computing" or "global computing", such as SETI@home [6], Folding@home [7], Prediction@home [8]. Without providing a programming model, they are just used to solve one scientific or commercial HTC problem. With its OMP programming model, $\mathrm{P} 2 \mathrm{HP}$ can solve a large range of problems.

Furthermore, a number of middleware for public-resource computing have been provided, such as BOINC [5], OmniRPC [9], and XtremWeb [10]. The clients of these systems contact directly with the server or through an agent, which is different from the communication mechanism of P2HP. With autonomous domains, the resource management of P2HP is decentralized, and most communication between entries exists in local domains.

OurGrid [11] is a solution of running applications of Bag-of-Tasks. The job scheduler is based on a provider-consumer algorithm. Different from the OMP programming model of $\mathrm{P} 2 \mathrm{HP}$, it provides a script-command application programming model which is limited for applications.

\section{System Architecture}

P2HP is constructed as a 3-layer network, as well as a pool for accessing data (Datapool) and a user interface (User), shown in Figure 1. The module User is used to manage the submission of applications, and Datapool is used to manage the storage of applications' related data.

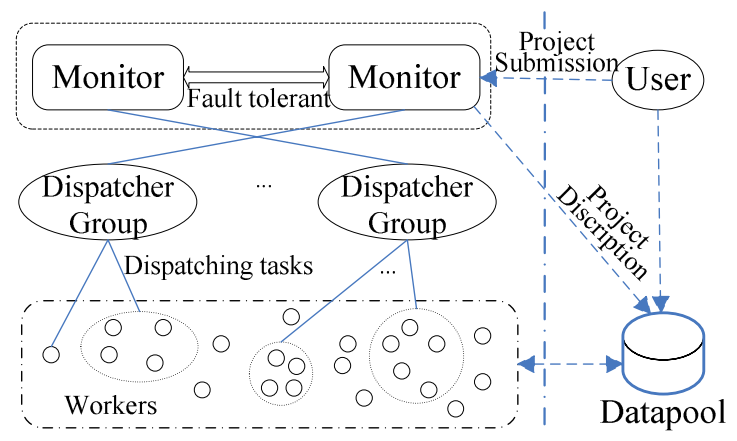

Fig. 1. Architecture of $\mathrm{P} 2 \mathrm{HP}$

The basic components of the 3-layer network are monitors, dispatchers and workers. The monitoring of the whole system is done by monitors, which are grouped as Monitor Group (MG). Dispatchers schedule tasks to the workers, and all of the dispatchers are grouped as Dispatcher Groups (DGs). A dispatcher can choose one or more monitors to join, and the chosen monitor is then its host monitor. Workers execute subtasks of applications and each of them is attached to a dispatcher. 
The parallelism of applications in P2HP is in the application level, and applications are divided into small tasks. Each task finishes certain computing work for the application. One of them participates in the control of the tasks' execution flow, and it is defined as the main task, while the others are defined as subtasks. Programmed with OMP, applications are transferred into Datapool, and a project file (JobFile) is generated, which is submitted to a monitor in the MG. They are then performed through the scheduling process of $\mathrm{P} 2 \mathrm{HP}$.

\section{Design Issues}

\subsection{Entries of 3-Layer Network}

The running of the whole system is controlled by entries of the 3-layer network. Monitors inquire the information of the actions of participants and the running states of applications. Dispatchers poll the attached workers to inquire their states, which is similar to the heartbeat mechanism. The worker accepts their messages and sends back state information for its available hardware and software resources and executes dispatched tasks.

A dispatcher with a number of attached workers forms an autonomous domain, and the dispatcher is the dominator of the domain. Each domain is constructed as an unstructured P2P network. All the dominators are monitored by the monitor. The metric with autonomic domains for resource management in the 3-layer network exploits the scalability of P2HP.

\subsection{Data Management}

Data is accessed in the Datapool, and all the requested data is transferred by a transmission protocol and managed by the local storage system.

\subsubsection{Storage Management}

In the Datapool, the data is accessed in the form of files, and they are assembled in a directory. The directory management of the Datapool is combined with a lightweighted database, the Berkeley DB, which is an embedded database. It is used to record and dynamically update the information of tasks' states.

Services are provided by the database, and they give universal interfaces to the Datapool's requests from clients. With the database turned on, the process of the usage for these services is passed through applying a service, attaining information and returning its result.

\subsubsection{Data Transmission}

The data transmission is implemented as a Fast Data Transfer Protocol (FDTP). It is based on a network-connected pipe, which consists of a message channel for messages and a data channel for corresponding data files.

During the transmission, the data files are formatted as an $\mathrm{I} / \mathrm{O}$ stream, and states are defined, such as BEGIN_TRANS and FINISH_ONCE for the beginning and finishing of the process, IDLE for none, SYN_RECEV and SYN_SENT for synchronization of receiving and sending a message. The transmission process is expressed by a state transition shown in Figure 2, where the real line represents the server's state transition, while the dashed one represents that of the clients. 


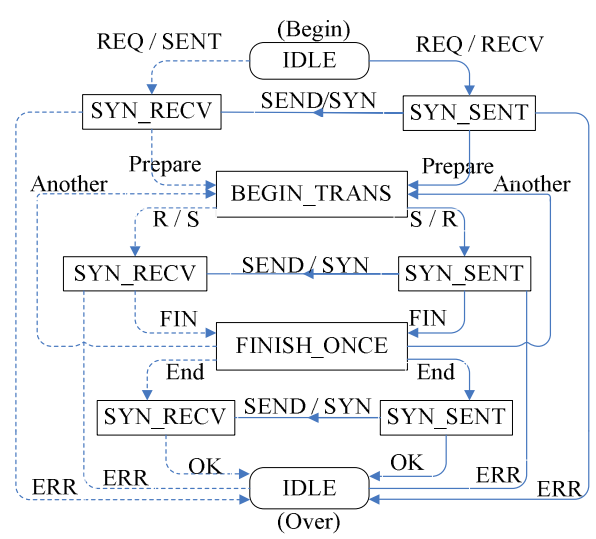

Fig. 2. State transition during data's transmission

Both clients and the server start the transmission from the IDLE state, and the transition of their states will pass through BEGIN_TRANS, EINISH_ONCE, and IDLE in the end. A file transfer starts with BEGIN_TRNAS, and ends with FINISH_ONCE. If there are other data files to be transferred, both server and client are BEGIN_TRANS again and begin to resolve the next data file. If an error occurs, the transferring fails and both of them are IDLE and prepare next transaction for next request. The procedure is iterated until all data files have been transferred, or an error occurs. Each transition will be synchronized with SYN. One of them is the sender, while the others are the receivers.

\subsection{Scheduling}

The running of an application is initiated by the main task, which applies subtasks from the monitor with a program ID and the number of subtasks. The Monitor selects one or several dispatchers with the lowest load and redirects the JobFile to them. Each of these dispatchers is assigned part of the project's subtasks. Then the main task requests those dispatchers to execute its subtasks.

The dispatcher schedules its workers to execute subtasks. If there are unfinished subtasks, the dispatcher distributes them to the idle workers. The worker who gets a task attains the task's program and related data from the Datapool. Then the worker performs the execution of subtasks. Finally, the worker returns the result of the subtask to the Datapool after finishing the execution. After all the subtasks have been finished, the main task gets their results from the Datapool, and further resolves them to get the final result.

\subsection{Monitoring and Fault Tolerance}

To ensure the availability, measures have been taken to trace P2HP's computing resources and the running states of applications' tasks. It is collaborated by monitors and dispatchers. The monitor is the supervision center of the system.

The monitor and the dispatcher cooperate as follows. In an autonomous domain, workers are traced by the dispatcher periodically. All the dispatchers are traced by the 
monitor. The transaction to each entry's fault is trigged off during the tracing. The load of the entry with errors will be substituted by another one in its group. This procedure is controlled by the entry in the top layer of network.

\subsection{Software Development Kit}

To be adaptive to multiple applications, the OMP programming model has been provided for users. OMP has been deliberately kept simple with minimum conceptual overhead. It consists of a communication library (ComLib) and a software development kit (SDK) for users.

The ComLib is used for the communication between tasks and the Datapool, and it is packaged based on the system's data transmission module. The SDK is responsible for providing application programming interfaces for subtasks (SubAPI) and the main tasks (MainAPI).

The OMP is a one-sided message passing model. As the shared remote space, the Datapool will respond to the request messages. When the client that has dispatched tasks needs the correlative data, it only locally triggers off a message like sending or receiving, and resolves this message to the Datapool. Then the requested data will be transferred by the ComLib between the Datapool and the client.

\section{Experiment}

To evaluate the performance of P2HP, we use a benchmark with speedup of the parallelized program in P2HP to the original sequential one in a PC. The benchmark is sequence alignment, which is a basic problem of bioinformatics.

\subsection{Methods}

The environment of P2HP is conducted in a network with 30 normal PCs. All computers are connected by a $100 \mathrm{Mbps}$ Ethernet switch. The frequencies of CPUs are from $1 \mathrm{GHz}$ to $2.4 \mathrm{GHz}$. The Datapool is installed in a PC, where the Monitor and the Dispatcher are also configured. Other computers join the platform as workers as necessary.

CLUSTAL W and its variants are the most common used software packages for multiple alignments. First, the sequences are aligned by the sequential CLUSTAL W program in a PC. Then, as presented in [3], the sequential one is parallelized with OMP and run in P2HP. Assume that the number of sequences for multiple alignments is $n$, and the number of pairwise alignments in a subtask is $k$. Then the number of the whole pairwise alignments $P_{A}$ and the number of subtasks $N_{S}$ are shown in Formula 1 and 2 . The phylogenetic tree is constructed according to the distance matrix of all the pairwise alignments, which is used to conduct the order of progressive alignment for multiple sequences.

$$
\begin{gathered}
P_{A}=\frac{n \times(n-1)}{2} \\
N_{S}=\frac{P_{A}}{k}=\frac{n \times(n-1)}{2 k}
\end{gathered}
$$




\subsection{Evaluation}

One hundred sequences are selected from NCBI (National Center for Biotechnology Information) which is one of the sequence databases in the world. The number of each sequence's letters is from 957 to 1534 . In the CLUSTAL W program, the formation of phylogenetic tree through pairwise alignments costs about 440s.

In the parallelized execution for pairwise alignments in $\mathrm{P} 2 \mathrm{HP}$, it is assumed that the time for each pairwise alignment is $C_{P}$, and the communication time is $C_{M}$. The time for disposing of distance matrix is $P_{T}$, and the execution time $T_{P}$ of the parallelized CLUSTAL W in P2HP is shown as Formula 3. The execution time $T_{S}$ for the sequential program is as Formula 4.

The speedup $V$ of the parallelized one in $\mathrm{P} 2 \mathrm{HP}$ to the sequential is depicted in Formula 5. Combined with Formula 1 and 2, $V$ is further represented in Formula 6. With the number of sequences as a constant, the speedup increases with the decreasing of $k$, and the maximum value is attained when $k=1$.

$$
\begin{gathered}
T_{P}=\frac{P_{A} \times\left(C_{P}+C_{M}\right)}{N_{S}}+P_{T} \\
T_{S}=P_{A} \times\left(C_{P}-E\right) \\
V=\frac{T_{S}}{T_{P}}=\frac{N_{S} \times\left(C_{P}-E\right)}{C_{P}+C_{M}+N_{S} \times P_{T} / P_{A}} \\
V=\frac{n \times(n-1)}{2 k} \times \frac{C_{P}-E}{C_{P}+C_{M}+P_{T} / 2 k}
\end{gathered}
$$

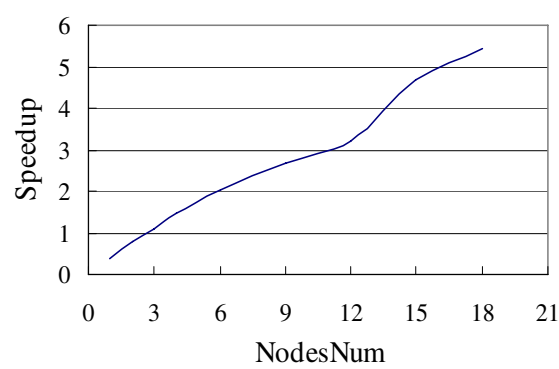

Fig. 3. Speedup with the increase of workers

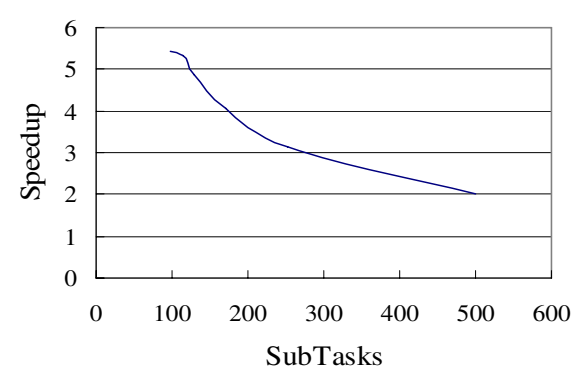

Fig. 4. Speedup with the increase of subtasks

To further verify its performance, we divide the alignments into 98 subtasks and increase the workers. The time for sequence alignment in P2HP decreases, and the speedup to the sequential CLUSTAL W increases, shown in Figure 3. If the number of workers is small, the speedup is less than 1 , which means that the parallel execution of a small quantity of workers can not afford the cost of the communication. 
Supposed that the number of workers is a constant as 18 and the number of subtasks is much larger than 18, the speedup to the sequential program is decreasing with the rise of the subtasks' number, as shown in Figure 4. It shows that P2HP is more adaptive to the computation intensive applications.

The experiment demonstrates that P2HP is an efficient and practical distributed HPC platform, especially to the computation intensive applications. To get the expected performance of P2HP, the number of subtasks should match that of workers in a limited environment, and applications should be divided into more subtasks if there are enough workers in the system.

\section{Conclusion}

P2HP is constructed as the 3-layer network that consists of monitors, dispatchers and workers, as well as a user and a Datapool. Based on unstructured P2P, P2HP is scalable and adaptive to dynamic environments. Augmenting the number of workers, its computing capability is increasing. With an easy configuration, P2HP is applicable. Moreover, the benchmark shows that it can provide scalable and enormous power for HPC applications.

\section{References}

[1] G. Bell and J. Gray, "What's next in high-performance computing", Communications of the ACM, 45 (2), pp.91 95, 2002.

[2] R. Raman, M. Livny, and M. Solomon, "Matchmaking: distributed resource management for high throughput computing", Proceedings of the Seventh IEEE International Symposium on High Performance Distribute Computing (HPDC'98), 1998.

[3] H. Jin, F. Luo, Q. Zhang, X. Liao, H. Zhang, "OMP: a one-sided message passing programming model for P2HP”, Proceedings of the Seventh International Meeting on High Performance Computing for Computational Science (VECPAR'06), 2006.

[4] V. Lo, D. Zappala, D. Zhou, Y. Liu, and S. Zhao, "Cluster computing on the fly: P2P scheduling of idle cycles in the Internet", Proceedings of the 3rd International Workshop on Peer-to-Peer Systems (IPTPS'04), 2004.

[5] D. P. Anderson, "BOINC: A system for public-resource computing and storage", Proceedings of the Fifth IEEE/ACM International Workshop on Grid Computing (GRID'04), pp.4-10, 2004.

[6] D. P. Anderson, J. Cobb, E. Korpela, M. Lebofsky, and D. Werthimer, "SETI@ home: An experiment in public-resource computing", Communications of the ACM, 45(11), pp.56-61, 2002.

[7] V. S. Pande, I. Baker, J. Chapman, S. P. Elmer, S. Khaliq, S. M. Larson, Y. M. Rhee, M. R. Shirts, C. D. Snow, E. J. Sorin, and B. Zagrovic, "Atomistic Protein Folding Simulations on the Submillisecond Time Scale Using Worldwide Distributed Computing", Biopolymers, Vol.68, pp.91-109, 2003.

[8] M. Taufer, C. An, A. Kerstens, and C. L. Brooks, "Predictor@Home: A 'protein structure prediction supercomputer' based on public-resource computing", Proceedings of the 19th IEEE International Parallel and Distributed Processing Symposium (IPDPS'05), 2005. 
[9] M. Sato, T. Boku, and D. Takahashi, "OmniRPC: A grid RPC system for parallel programming in cluster and grid environment", Proceedings of 3rd International Symposium on Cluster Computing and the Grid (CCGrid'03), pp.206-213, 2003.

[10] G. Fedak, C. Germain, V. Neri, and F. Cappello, "XtremWeb: A generic global computing system", Proceedings of the 1st IEEE/ACM International Symposium on Cluster Computing and the Grid (CCGrid'01), 2001, pp.582-587, 2001.

[11] N. Andrade, W. Cirne, F. Brasileiro, and P. Roisenberg, "OurGrid: an approach to easily assemble grids with equitable resource sharing", Proceedings of the 9th Workshop on Job Scheduling Strategies for Parallel Processing (JSSPP'03), pp.61-86, 2003. 